\title{
Neuroeconomía y toma de decisiones financieras: aproximación desde una revisión sistemática de literatura *
}

\author{
Neuroeconomics and Financial Decision-Making: A Systematic Literature \\ Review Exploring the Field
}

\author{
Daniel Osorio-Barreto \\ Magíster en Economía, Universidad Autónoma de Manizales, \\ Manizales - Colombia, dosoriob@autonoma.edu.co \\ Daniel Alfredo Landínez-Martínez \\ Magíster en Neuropsicología, Universidad Católica Luis Amigó, \\ Manizales - Colombia, daniel.landinezma@amigo.edu.co \\ Juan Carlos Chica-Mesa \\ Doctor en Industria y Organizaciones, Universidad Nacional de Colombia, \\ Manizales - Colombia, jcchicam@unal.edu.co
}

\begin{abstract}
Cómo citar / How to cite
Osorio-Barreto, D., Landínez-Martínez, D. A., Chica-Mesa, J. C. (2022). Neuroeconomía y toma de decisiones financieras: aproximación desde una revisión sistemática de literatura. Revista CEA, v. 8, n. 16, e1911.

https://doi.org/10.22430/24223182.1911
\end{abstract}

Recibido: 31 de mayo de 2021

Aceptado: 13 de septiembre de 2021

\section{Resumen}

El estudio de la toma de decisiones financieras es un campo emergente de investigación básica y aplicada. Frente a este panorama, los avances en el conocimiento del funcionamiento cognitivo permiten generar la pregunta de cómo, desde la neuroeconomía, se analiza la toma de decisiones financieras. En este sentido, el presente trabajo tuvo como objetivo principal analizar estudios relacionados sobre neuroeconomía, haciendo énfasis en aquellos enfocados a comprender la toma de decisiones financieras. Para ello se realizó una revisión sistemática de literatura soportada en la base de datos Web of Science para identificar las principales referencias sobre el tema, teniendo en cuenta su tipo de publicación, autores, área de conocimiento, palabras clave, enfoque e instrumentos

\footnotetext{
* Este artículo se deriva de la tesis doctoral «Ensayos en macroeconomía del comportamiento: expectativas de inflación y sus determinantes», y ha sido financiado con recursos de la Universidad Autónoma de Manizales.
} 
utilizados. Con base en la estrategia metodológica propuesta, se identificaron diferentes trabajos que han analizado la toma de decisiones financieras desde otras perspectivas: riesgo financiero, finanzas personales, decisiones de inversión, entre otros, siendo el diseño de experimentos, apoyado por imágenes diagnósticas, los estudios de mayor impacto. Finalmente, la revisión sistemática encuentra que los estudios de alto impacto se ubican en Estados Unidos y Europa con una ampliación a lo largo del tiempo de técnicas empíricas y experimentales para comprender el proceso de toma de decisiones financieras; adicionalmente, esta revisión pretende ser referente de subsiguientes investigaciones relacionadas en América Latina.

Palabras clave: economía del comportamiento, neuroeconomía, toma de decisiones, finanzas comportamentales, racionalidad limitada.

Clasificación JEL: D01, D90, D91, G41.

\title{
Highlights
}

- La economía del comportamiento y la neuroeconomía se han erigido como programas de investigación científica potentes y en ascenso para el abordaje de fenómenos económicos.

- Los principales trabajos sobre neuroeconomía aplicados a la toma de decisiones financieras están asociados a diferentes campos de análisis como perfil de riesgo, decisiones de inversión, confianza, entre otros.

- La neuroeconomía se constituye en un tema de investigación emergente en países latinoamericanos.

\begin{abstract}
Financial decision-making is an emerging field of study in basic and applied research. Nevertheless, advances in the knowledge of cognitive functioning have questioned the way neuroeconomics analyzes this type of decision-making. This paper reviews the literature on neuroeconomics, specifically the studies focused on understanding financial decision-making. This systematic literature review was conducted using the Web of Science database to identify the most important publications on the topic, considering the type of publication, authors, knowledge field, keywords, approach, and instruments used. The methodology proposed here retrieved a set of documents that have analyzed financial decision-making from other perspectives (i.e., financial risk, personal finance, and investment decisions, among others). The most influential studies have implemented design of experiments supported by diagnostic imaging. Finally, this systematic literature review found that most high-impact papers have been published in the United States and Europe; in addition, empirical and experimental techniques have long been used to understand financial decision-making. This review aims to be a reference point for subsequent research in this field in Latin America.
\end{abstract}

Keywords: Behavioral economics, neuroeconomics, decision-making, behavioral finance, limited rationality.

JEL classification: D01, D90, D91, G41. 


\section{Highlights}

- Behavioral economics and neuroeconomics have become powerful scientific research agendas that advance toward the study of economic phenomena.

- The most important publications on neuroeconomics applied to financial decision-making analyze aspects such as risk profile, investment decisions, and trust, among others.

- Neuroeconomics is an emerging research topic in Latin American countries.

\section{INTRODUCCIÓN}

El estudio de la toma de decisiones financieras es un campo emergente de investigación básica y aplicada. Frente a este panorama, los avances en el conocimiento del funcionamiento cognitivo permiten generar la pregunta de cómo, desde la neuroeconomía, se analiza la toma de decisiones financieras. Para abordarlo, se ha propuesto como objetivo general analizar estudios relacionados sobre neuroeconomía, haciendo énfasis en aquellos enfocados a comprender la toma de decisiones financieras y como objetivos específicos los siguientes: 1) sintetizar los elementos teóricos esenciales de la neuroeconomía que permitan una mayor comprensión de sus principales trabajos; 2) clasificar los principales trabajos sobre neuroeconomía relacionados con la toma de decisiones financieras según los autores más relevantes, revistas destacadas, tema central de interés y enfoque de los trabajos; 3) identificar el avance de la investigación sobre neuroeconomía en América Latina y en Colombia.

La discusión sobre el impacto del comportamiento racional de los individuos y su incidencia en las relaciones entre las personas, quienes se configuran en agentes económicos, ha dado lugar al surgimiento de un campo de investigación denominado «economía del comportamiento» (behavioral economics), área de la cual se deriva la neuroeconomía, que pretende estudiar el comportamiento de los individuos, teniendo en cuenta su racionalidad limitada y los fallos que pueden presentarse si se supone una racionalidad completa a la hora de anticipar posiciones/acciones en escenarios intertemporales (Srivastava et al., 2019).

En este sentido, Angner y Loewenstein (2012) y Martin y Sbicca, (2021) afirman que la economía del comportamiento representa un cambio de rumbo frente al mainstream propuesto por la economía neoclásica a la hora de comprender el proceso de elección de los individuos (significativamente influido por el conductismo, el hedonismo, el verificacionismo y el operacionalismo), buscando incrementar el poder explicativo y predictivo de la teoría económica de la mano de la psicología, incluyendo en el análisis entidades no observables como las emociones y la heurística.

Según Angner y Loewenstein, (2012) y Carminati, (2020), diferentes escuelas del pensamiento económico, iniciando desde los clásicos, aportaron a la reflexión de la economía comportamental, al plantear diversos interrogantes frente al proceso de toma de decisiones de los agentes económicos y su conducta. En este sentido, según los autores mencionados, la economía del comportamiento se erige como área de conocimiento con los aportes de Katona $(1946,1953)$ y Simon $(1955,1989)$ al reflexionar, de la mano de la psicología, sobre la racionalidad limitada de las personas, al cuestionarse sobre los supuestos tradicionales de la economía, abriendo la discusión para trabajos que se constituyeron como seminales en la consolidación de este programa de investigación científica. 
Específicamente, como trabajos seminales de la neuroeconomía se destacan los aportes de Kahneman y Tversky (1979) y Tversky y Kahneman (1991), quienes proponen una teoría prospectiva, cuestionando los supuestos de la teoría de la utilidad esperada propuesta por Von Neumann y Morgenstern (1944), ajustada a sus procesos cognitivos. Relacionada con esta teoría, Thaler (1980) cuestiona las teorías positivas y normativas que analizan cómo los consumidores deben elegir en un modelo racional de maximización de utilidad, y agrega que estos modelos descuidan cómo los consumidores eligen actuando, en ocasiones de manera inconsistente, frente a los postulados tradicionales que no tienen en cuenta los siguientes aspectos: subvaloración de los costos de oportunidad, fallo al ignorar costos hundidos, autocontrol, entre otros.

Ampliando el análisis de la toma de decisiones, Loewenstein (1996) y Camerer (2013) afirman que esta es un área de investigación donde convergen la economía y la psicología cognitiva, teniendo en cuenta que elementos como los factores viscerales (hambre, sed, deseo sexual y sueño, entre otros) y la interdependencia estratégica inciden sobre esta, comprendiendo la racionalidad limitada, no como la violación del supuesto de transitividad e independencia, sino como un proceso de percepción subjetiva que depende de múltiples factores que condicionan la elección (emociones, factores viscerales, influencia social, etc.).

Frente a la consolidación de la neuroeconomía, es pertinente destacar las definiciones de Glimcher (2003), Angner y Loewenstein (2012) y Bermejo et al. (2011), relacionada con el estudio, a partir de herramientas desarrolladas por los neurocientíficos, de cómo interactúa el cerebro humano (específicamente los fundamentos neuronales) con su entorno para generar un comportamiento económico. Así, gran parte sus estudios están basados en el escaneo del cerebro de sujetos que participan en experimentos mientras realizan tareas asociadas a la economía del comportamiento, tales como juego del ultimátum, toma de decisiones bajo riesgo e incertidumbre, elección intertemporal, disyuntiva en compra de bienes, entre otros.

Finalmente, para comprender los elementos neurofisiológicos, neuroestructurales y neurocognitivos que subyacen el comportamiento humano frente a la toma de decisiones financieras es importante resaltar que la corteza prefrontal consta de tres regiones principales que incluye la corteza orbitofrontal, la corteza cingulada anterior, y la corteza prefrontal dorsolateral, que interactúan entre sí, así como con estructuras subcorticales, tales como los ganglios basales, tálamo, y cerebelo, e influyen en el proceso de toma de decisiones (Bigorra et al., 2016). La corteza orbitofrontal tiene conexiones límbicas y es fundamental en la toma de decisiones basada en emociones y en recompensas. La corteza prefrontal dorsolateral y la corteza cingulada anterior facilitan las decisiones intelectuales que dependen de la memoria de trabajo y el razonamiento (Satterfield y Killgore, 2019; Yang et al., 2017).

De la misma manera, la corteza orbitofrontal está funcionalmente organizada de tal manera que la porción medial monitorea y decodifica las recompensas, mientras que la porción lateral evalúa el castigo. El valor de la recompensa por factores reforzadores primarios concretos, tales como el tacto y el gusto, son codificados en la corteza orbitofrontal posterior, mientras que el valor de factores reforzadores secundarios más complejos, como el dinero, son codificados en la corteza orbitofrontal anterior (Fairchild et al., 2009). En este sentido, los pacientes con lesiones en la corteza orbitofrontal tienen dificultades para rechazar un estímulo a pesar de estar relacionado con consecuencias negativas. De la misma manera, estos pacientes tienen dificultades para ejecutar tareas que 
requieren empatía o teoría de la mente (TOM), fallando en el procesamiento y reconocimiento de las emociones de otros, y manifiestan alteración del juicio en contextos sociales (Zegarra-Valdivia y Chino, 2018). Tales limitaciones en el aprendizaje social e identificación de las emociones frecuentemente conllevan a un síndrome clínico relacionado con la desinhibición, impulsividad, aumento de las conductas de toma de riesgo y la incapacidad para modificar la conducta a pesar de consecuencias sociales negativas (Fatima et al., 2020).

\section{METODOLOGÍA}

A partir de la discusión anterior, se realiza una revisión sistemática de literatura basada en una ecuación de búsqueda (EB) donde se identifican tres descriptores claves que se plantean de manera conjunta (búsqueda realizada en inglés y español): i) neuroeconomía (neuroeconomics), teniendo en cuenta que sus instrumentos de análisis y medición han generado un avance sustancial en la economía del comportamiento, ii) decision* (decisiones), con el fin de rastrear estudios que estén asociados a la toma de decisiones, iii) financ*, buscando que con este prefijo se pueda encontrar cualquier asociación con aspectos financieros. La Figura 1 representa la EB propuesta. Cabe aclarar que el asterisco $(*)$ se utiliza en las palabras indicadas para indicar en los motores de búsqueda la inclusión de todas las combinaciones posibles de sufijos.

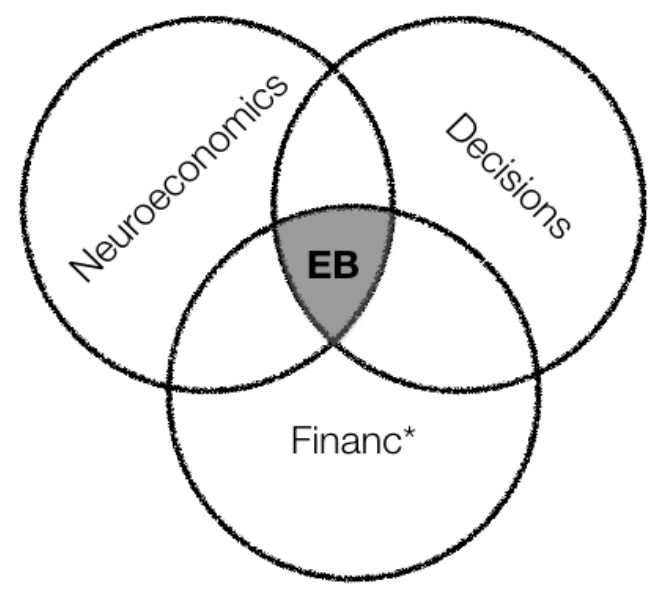

EB $=$ Neuroeconomics AND Decisions AND Financ*

Figura 1. Esquema ecuación de búsqueda (EB)

Figure 1. Scheme of topics in the search string Fuente: elaboración propia.

Una vez planteada esta ecuación de búsqueda, se resalta que el alcance de esta revisión sistemática de literatura es la aplicación de la EB en la base de datos Web of Science (WoS), revisando los estudios disponibles hasta el año 2019 bajo el criterio de tópico (que incluye título, abstract y palabras clave).Cabe mencionar que, dado el objetivo general propuesto relacionado con analizar estudios relacionados sobre neuroeconomía haciendo énfasis en aquellos enfocados a comprender la toma de decisiones financieras, las palabras claves de la EB son amplias en el sentido que pretende dar al lector un panorama general del estado de conocimiento en el área y sus diferentes campos de 
conocimiento acorde a la base de datos WoS. Basado en los objetivos propuestos, se realiza un rastreo de las referencias asociadas a la EB para, después, analizar detalladamente los principales elementos de los trabajos hallados tales como palabras clave, tema central de investigación, enfoques y herramientas más relevantes.

\section{RESULTADOS}

En esta sección se hizo una revisión de los 54 trabajos identificados con el planteamiento de la EB. En primer lugar, en la Figura 2, se observa el posicionamiento del área de análisis en términos del número de publicaciones y citaciones.

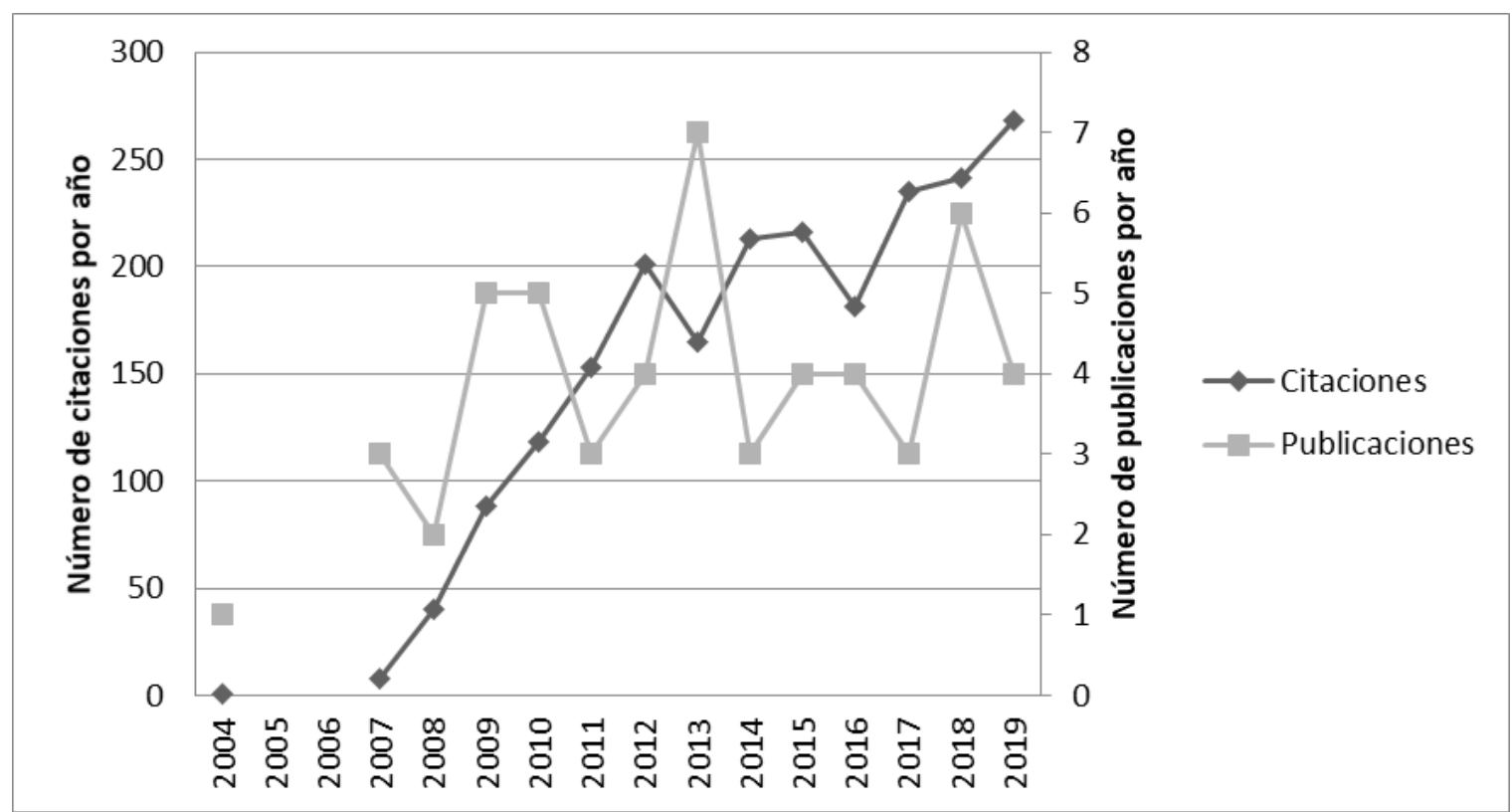

Figura 2. Publicaciones y citaciones por año según WoS

Figure 2. Annual number of publications and citations according to WoS Fuente: elaboración propia.

Adicional a la figura anterior, se observó que existe una gran variedad de áreas temáticas, categorías definidas por WoS, que investigan sobre neuroeconomía, partiendo del hecho del carácter multidisciplinar de las publicaciones: neurociencias (38.89\%), economía (18.52\%), ciencias multidisciplinarias (11.11\%), psicología (9.26\%), entre otros.

De otro lado, frente a los autores, la Figura 3 plantea el ranking acorde a su producción científica a lo largo del período de tiempo analizado (el tamaño del círculo es proporcional al número de artículos por año y la tonalidad de la escala de grises varía según el número de citaciones por año). Para el caso de la EB, se destacaron como autores relevantes en el campo P. Tobler, P. Bossaerts, M. Baddeley y C. Camerer. 


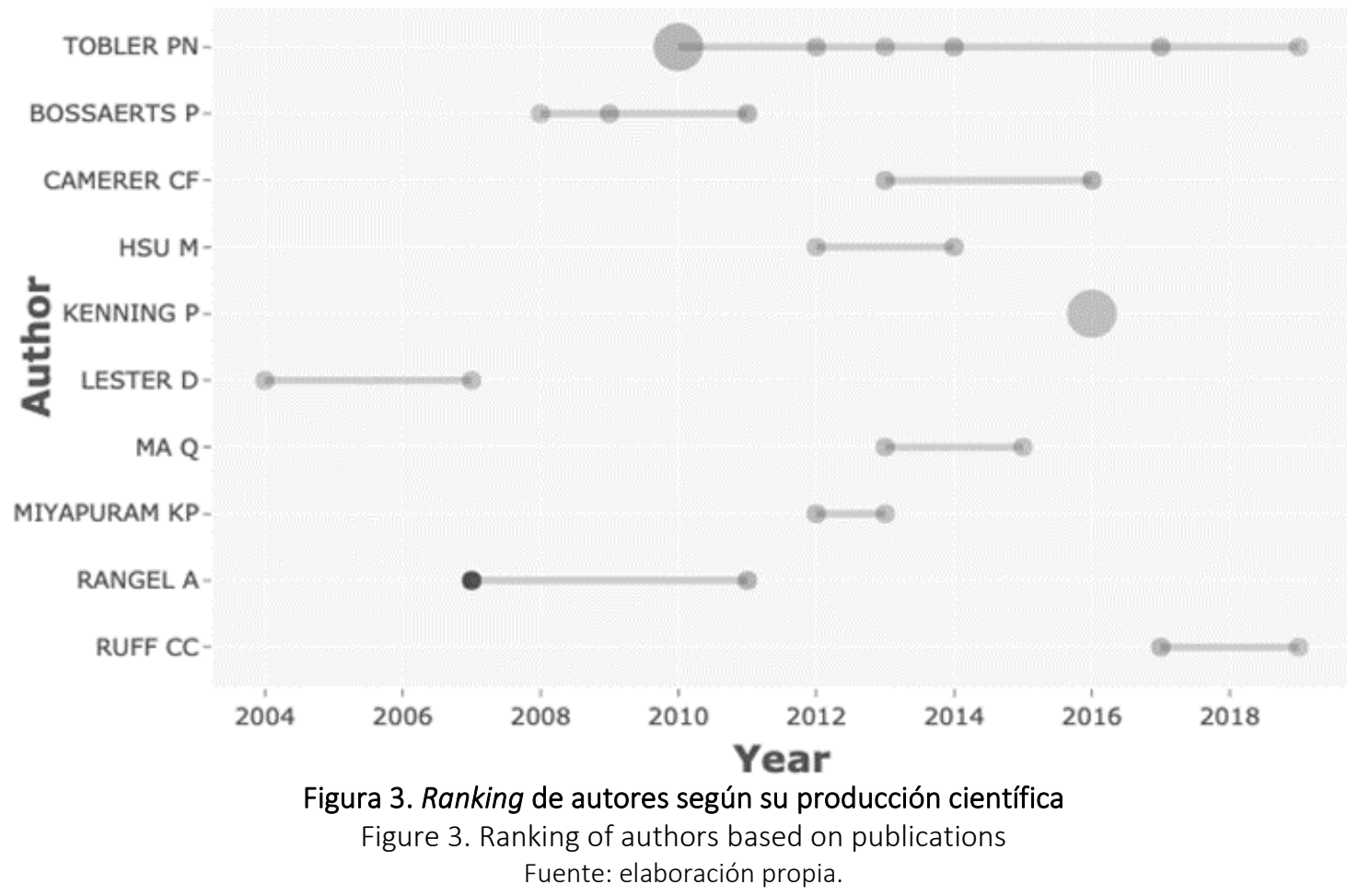

En relación con la distribución de las publicaciones según su país, basado en el análisis bibliométrico desarrollado por Aria y Cuccurullo (2017), se encontró que los países más productivos (en términos de la procedencia de los autores) son Estados Unidos (19 publicaciones), Reino Unido (6 publicaciones), Alemania (5 publicaciones) y China (4 publicaciones). De estos resultados obtenidos se destacó que no se registran casos de aplicación para América Latina (cuando se realiza un rastreo preliminar por Google Académico se encuentran diversas aplicaciones para el caso del neuromarketing y discusiones teóricas con cortas aplicaciones para la neuroeconomía en revistas que no están indexadas en las bases de datos analizadas), lo cual potencia el interés de ahondar en esta temática.

De manera complementaria a los análisis anteriores, es pertinente analizar el ranking de los artículos con mayor número de citaciones para la EB propuesta, con el fin de identificar publicaciones estratégicas para su estudio detallado. En la Tabla 1 se presenta el ranking de los diez artículos con mayor citación, basados en la base de datos de WoS. 
Tabla 1. Ranking de los diez artículos con mayor citación

Table 1. Top ten most-cited articles

\begin{tabular}{|c|c|c|c|c|c|}
\hline Posición & Título & Autores & Revista & $\begin{array}{c}\text { Año } \\
\text { publicación }\end{array}$ & $\begin{array}{c}\text { Total } \\
\text { citaciones }\end{array}$ \\
\hline 1 & $\begin{array}{l}\text { Orbitofrontal cortex encodes } \\
\text { willingness to pay in everyday } \\
\text { economic transactions }\end{array}$ & Plassmann et al. & $\begin{array}{l}\text { Journal of } \\
\text { Neuroscience }\end{array}$ & 2007 & 459 \\
\hline 2 & $\begin{array}{l}\text { Endogenous steroids and } \\
\text { financial risk taking on a } \\
\text { London, trading floor }\end{array}$ & Coates y Herbert & $\begin{array}{l}\text { Proceedings of } \\
\text { the National } \\
\text { Academy of } \\
\text { Sciences of the } \\
\text { United States } \\
\text { of America }\end{array}$ & 2008 & 298 \\
\hline 3 & $\begin{array}{c}\text { Differential encoding of losses } \\
\text { and gains in the human } \\
\text { striatum }\end{array}$ & Seymour et al. & $\begin{array}{l}\text { Journal of } \\
\text { Neuroscience }\end{array}$ & 2007 & 280 \\
\hline 4 & $\begin{array}{l}\text { Gender differences in financial } \\
\text { risk aversion and career } \\
\text { choices are affected by } \\
\text { testosterone }\end{array}$ & Sapienza et al. & $\begin{array}{l}\text { Proceedings of } \\
\text { the National } \\
\text { Academy of } \\
\text { Sciences of the } \\
\text { United States } \\
\text { of America } \\
\end{array}$ & 2009 & 270 \\
\hline 5 & $\begin{array}{l}\text { The Influence of Affect on } \\
\text { Beliefs, Preferences, and } \\
\text { Financial Decisions }\end{array}$ & $\begin{array}{l}\text { Kuhnen y } \\
\text { Knutson }\end{array}$ & $\begin{array}{c}\text { Journal of } \\
\text { Financial and } \\
\text { Quantitative } \\
\text { Analysis } \\
\end{array}$ & 2011 & 91 \\
\hline 6 & $\begin{array}{l}\text { Herding, social influence and } \\
\text { economic decision-making: } \\
\text { socio-psychological and } \\
\text { neuroscientific analyses }\end{array}$ & Baddeley & $\begin{array}{l}\text { Philosophical } \\
\text { Transactions of } \\
\text { the Royal } \\
\text { Society B- } \\
\text { Biological } \\
\text { Sciences } \\
\end{array}$ & 2010 & 66 \\
\hline 7 & $\begin{array}{c}\text { The somatic marker } \\
\text { framework as a neurological } \\
\text { theory of decision-making: } \\
\text { Review, conceptual } \\
\text { comparisons, and future } \\
\text { neuroeconomics research }\end{array}$ & $\begin{array}{l}\text { Reimann y } \\
\text { Bechara }\end{array}$ & $\begin{array}{l}\text { Journal of } \\
\text { Economic } \\
\text { Psychology }\end{array}$ & 2010 & 65 \\
\hline 8 & $\begin{array}{l}\text { Neuroeconomics and aging: } \\
\text { Neuromodulation of economic } \\
\text { decision making in old age }\end{array}$ & Mohr et al. & $\begin{array}{c}\text { Neuroscience } \\
\text { and } \\
\text { Biobehavioral } \\
\text { Reviews } \\
\end{array}$ & 2010 & 57 \\
\hline 9 & $\begin{array}{c}\text { Expert Financial Advice } \\
\text { Neurobiologically "Offloads" } \\
\text { Financial Decision-Making } \\
\text { under Risk }\end{array}$ & Engelmann et al. & PLOS ONE & 2009 & 52 \\
\hline 10 & $\begin{array}{c}\text { MAOA-L carriers are better at } \\
\text { making optimal financial } \\
\text { decisions under risk }\end{array}$ & Frydman et al. & $\begin{array}{l}\text { Proceedings of } \\
\text { the Royal } \\
\text { Society B- } \\
\text { Biological } \\
\text { Sciences }\end{array}$ & 2011 & 48 \\
\hline
\end{tabular}

Fuente: elaboración propia. 
Ahora bien, frente al tipo de publicación de los trabajos, se destacó que $90.74 \%$ corresponden a artículos científicos (49 publicaciones) y $9.26 \%$ a capítulos de libro (5 publicaciones). Específicamente, frente a los artículos científicos, las revistas con más publicaciones se resumen en la Tabla 2.

Tabla 2. Ranking de revistas según frecuencia de publicación

Table 2. Journal ranking based on publication frequency

\begin{tabular}{ccccc}
\hline Revista & Artículos & Área relacionada & Cuartil & Índice h \\
\hline $\begin{array}{c}\text { Proceedings of the National Academy of } \\
\text { Sciences of the United States of America }\end{array}$ & 4 & Multidisciplinaria & Q1 & 699 \\
\hline Neuroimage & 3 & Neurociencia cognitiva & Q1 & 320 \\
\hline Frontiers in Human Neuroscience & 2 & Neurociencia conductual & Q2 & 87 \\
\hline Frontiers in Neuroscience & 2 & Neurociencia (miscelánea) & Q1 & 71 \\
\hline Frontiers in Psychology & 2 & Psicología (miscelánea) & Q1 & 81 \\
\hline International Journal of Neuroscience & 2 & Neurociencia (miscelánea) & Q3 & 57 \\
\hline Journal of Economic Psychology & 2 & Economía y Econometría & Q2 & 82 \\
\hline Journal of Neuroscience & 2 & Neurociencia (miscelánea) & Q1 & 422 \\
\hline PloS ONE & 2 & Medicina (miscelánea) & Q1 & 268 \\
\hline
\end{tabular}

Fuente: elaboración propia a partir de WoS, Aria y Cuccurullo, (2017) y Scimago Journal \& Country Rank.

Adicionalmente, se resalta el análisis realizado de las palabras claves (keywords) donde se destaca como descriptores más comunes los asociados a neuroeconomics y decision-making, seguido por reward, fMRI, risk, emotions. El propósito de este rastreo es contemplar la pluralidad de conceptos asociados a la realización de investigaciones en el área de la neuroeconomía. La Figura 4 muestra las palabras clave (en inglés, para conservar la coherencia con las bases de datos) encontradas en la revisión realizada.

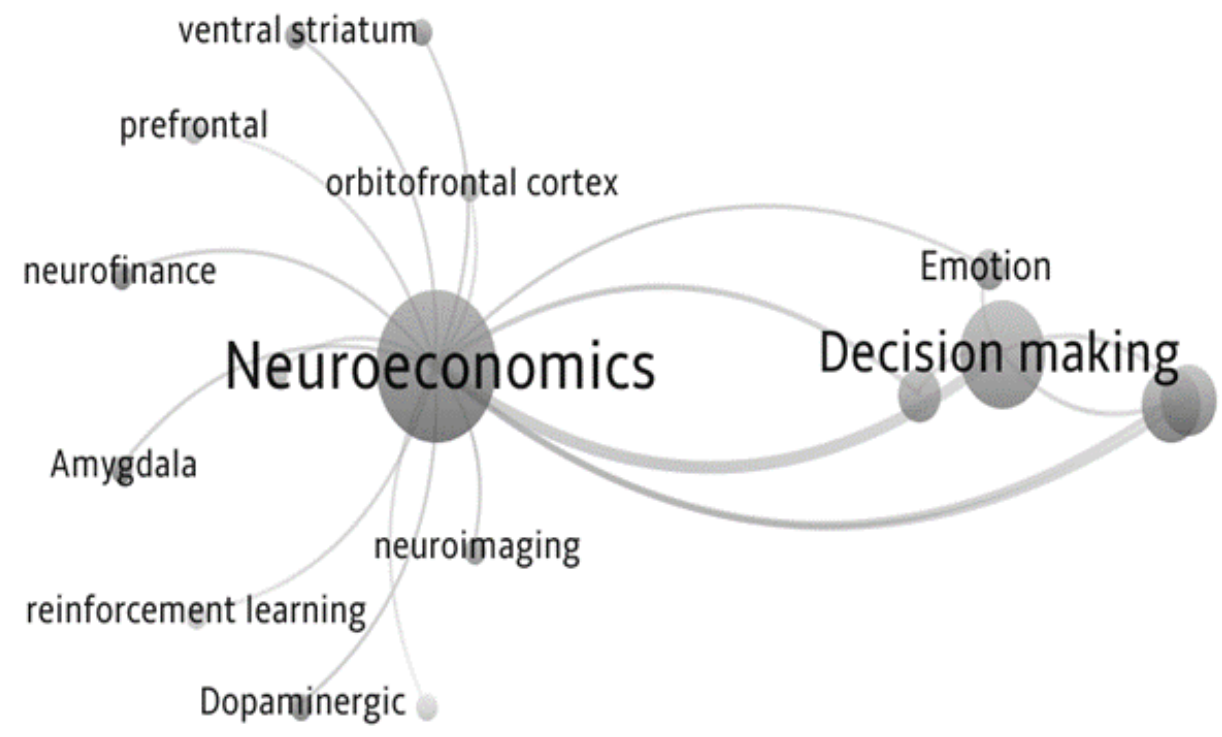

Figura 4. Gráfico de coocurrencias según EB

Figure 4. Network of co-occurrences found with the search string Fuente: elaboración propia a partir de Web of Science y la aplicación VOSviewer. 
Entrando en detalle a la revisión de artículos de la EB, con la herramienta Tree Of Science (ToS), desarrollada por Robledo et al. (2014), se identificaron los trabajos seminales (raíz), estructurales (tronco) y derivados o recientes (ramas) en la Tabla 3.

Tabla 3. Clasificación de artículos con base en la herramienta ToS

Table 3. Classification of articles by ToS

\begin{tabular}{cc}
\hline \multicolumn{1}{c}{ Clasificación } & \multicolumn{1}{c}{ Artículos } \\
\hline & Spinella et al. (2004), Spinella et al. (2007), Preuschoff et al. (2008), \\
& Bossaerts (2009), Engelmann et al. (2009), Halko et al. (2009), Sapienza et \\
& al. (2009), Burke et al. (2010), Kalenscher et al. (2010), Mohr et al. (2010), \\
& Bermejo et al. (2011), Frydman et al. (2011), Miyapuram et al. (2012), \\
& Shimokawa et al. (2012), Shoji y Kanehiro (2012), Zhu et al. (2012), Camerer \\
& (2013), Causse et al. (2013), Mullett y Tunney (2013), Pulcu et al. (2013), \\
& Shen et al. (2013), Tzieropoulos (2013), Zandstra et al. (2013), Chiong et al. \\
& (2014), Holper et al. (2014), Prinz et al. (2014), Apicella et al. (2015), Lucarelli \\
Recientes (ramas) & et al. (2015), Kolev et al. (2015), Wang (2015), Brunnlieb et al. (2016), Knecht \\
& y Kenning (2016), Suzuki et al. (2016), Mateu et al. (2017), Nienhusser y \\
& Oshio (2017), Raggetti et al. (2017), Saposnik et al. (2017), Ardalan (2018), \\
& Erkut et al. (2018), Harris et al. (2018), James (2018), Zhang (2018), Brisset y \\
& Jullien (2020), Hu y Shealy (2019), Saposnik et al. (2019), Srivastava et al. \\
& (2019) \\
\hline Plassmann et al. (2007), Seymour et al. (2007), Coates y Herbert (2008), \\
Zhong et al. (2009), Baddeley (2010), Reimann y Bechara (2010), Kuhnen y \\
Knutson (2011), Strombach et al. (2016)
\end{tabular}

Una vez analizados los trabajos encontrados por la EB, se realizó una clasificación de estos según el tema central de análisis, el enfoque del estudio y la principal herramienta utilizada; para ello, los esquemas que se presentan a continuación están basados en la estructura de presentación de la revisión sistemática de literatura realizada por Witte y López-Torres (2017).

En primer lugar, la Tabla 4 muestra la clasificación de los documentos revisados según su tema central de análisis, basado en categorías creadas de manera discrecional de tal manera que capture la esencia de cada estudio: 1) toma de decisiones, con el fin de rastrear estudios que iluminen sobre la aplicación de la neuroeconomía para resolver problemas asociados a este tema; 2) neuroeconomía, dirigido especialmente a la identificación de estudios que hayan hecho revisión de literatura o discusión teórica sobre este tema; 3) finanzas, que abarca estudios que analizaron diferentes aspectos de esta área como riesgo, gestión de finanzas personales, sistemas de inversión, entre otros; 4) conducta y otros estudios relacionados, la cual abarca diferentes escenarios de comportamiento de los agentes económicos con diferentes herramientas utilizadas y algunos estudios que analizaron aspectos puntuales de la salud humana como el envejecimiento, la obesidad y la modulación hormonal. 
Tabla 4. Clasificación de los trabajos según tema central de análisis Table 4. Classification of articles by main topic of analysis

\begin{tabular}{|c|c|c|}
\hline Tema central & Subtema & Trabajos \\
\hline \multirow{3}{*}{$\begin{array}{l}\text { Toma de } \\
\text { decisiones }\end{array}$} & General & $\begin{array}{c}\text { Plassmann et al. (2007), Seymour et al. (2007), Baddeley (2010), } \\
\text { Kalenscher et al. (2010), Mohr et al. (2010), Reimann \& Bechara } \\
\text { (2010), Causse et al. (2013), Mullett y Tunney (2013), } \\
\text { Tzieropoulos (2013), Lucarelli et al. (2015), Saposnik et al. } \\
\text { (2017), Hu y Shealy (2019) }\end{array}$ \\
\hline & Financieras & $\begin{array}{c}\text { Bossaerts (2009), Engelmann et al. (2009), Bermejo et al. (2011), } \\
\text { Frydman et al. (2011), Kuhnen y Knutson (2011), Holper et al. } \\
\text { (2014), Prinz et al. (2014), Mateu et al. (2017), Nienhusser y } \\
\text { Oshio (2017), Erkut et al. (2018) }\end{array}$ \\
\hline & Social & Zhu et al. (2012) \\
\hline \multicolumn{2}{|c|}{ Neuroeconomía } & Camerer (2013), Kolev et al. (2015), Srivastava et al. (2019) \\
\hline \multirow{7}{*}{ Finanzas } & Riesgo financiero & $\begin{array}{c}\text { Coates y Herbert (2008), Sapienza et al. (2009), Zhong et al. } \\
\text { (2009), Apicella et al. (2015), Zhang (2018) }\end{array}$ \\
\hline & Uso tarjetas de crédito & Spinella et al. (2004) \\
\hline & $\begin{array}{c}\text { Gestión de finanzas } \\
\text { personales }\end{array}$ & Spinella et al. (2007) \\
\hline & $\begin{array}{l}\text { Comportamiento } \\
\text { financiero }\end{array}$ & James (2018) \\
\hline & Errores financieros & Chiong et al. (2014) \\
\hline & $\begin{array}{c}\text { Recompensas } \\
\text { monetarias }\end{array}$ & Miyapuram et al. (2012) \\
\hline & $\begin{array}{c}\text { Teoría de } \\
\text { portafolio/Mercado de } \\
\text { valores }\end{array}$ & $\begin{array}{l}\text { Preuschoff et al. (2008), Shimokawa et al. (2012), Wang (2015), } \\
\text { Raggetti et al. (2017), Ardalan (2018), Brisset y Jullien (2020) }\end{array}$ \\
\hline \multirow{9}{*}{$\begin{array}{c}\text { Conducta y otros } \\
\text { estudios } \\
\text { relacionados }\end{array}$} & $\begin{array}{c}\text { Decisiones del } \\
\text { consumidor }\end{array}$ & Shoji y Kanehiro (2012), Zandstra et al. (2013) \\
\hline & Respuesta empática & Shen et al. (2013) \\
\hline & Emociones & Pulcu et al. (2013), Saposnik et al. (2019) \\
\hline & Contagio conductual & Burke et al. (2010), Suzuki et al. (2016) \\
\hline & $\begin{array}{l}\text { Comportamiento de } \\
\text { salud }\end{array}$ & Knecht y Kenning (2016) \\
\hline & $\begin{array}{l}\text { Comportamiento } \\
\text { motivado }\end{array}$ & Strombach et al. (2016) \\
\hline & Cooperación & Brunnlieb et al. (2016) \\
\hline & $\begin{array}{l}\text { Competencia entre } \\
\text { pares }\end{array}$ & Halko et al. (2009) \\
\hline & Salud pública & Harris et al. (2018) \\
\hline
\end{tabular}

En segundo lugar, se procedió a clasificar los estudios revisados en la EB según el enfoque de los mismos, planteando cuatro criterios de clasificación: 1) experimentos, los cuales se subdividen según el instrumento o herramienta relevante para el análisis del comportamiento de los sujetos (tales como resonancia magnética, electroencefalograma, pruebas de saliva, entre otros); 2) método 
cuantitativo, que recoge algunos artículos que, para comprobar sus hipótesis, se apoyan en el modelamiento estadístico y econométrico tradicional; 3) revisión de literatura, sección que recoge los trabajo que realizan un rastreo teórico a partir de revisiones de literatura, sistemáticas y no sistemáticas, que contextualizan los avances en los campos de estudio; 4) corta comunicación, que menciona un estudio arrojado por las bases de datos que plantea los elementos esenciales de su proyecto (y que no podría ubicarse en los campos anteriores). La Tabla 5 muestra la clasificación mencionada.

Tabla 5. Clasificación de los trabajos según enfoque del estudio e instrumento relevante Table 5. Classification of articles by research approach and relevant instrument

\begin{tabular}{|c|c|c|}
\hline Enfoque & Instrumento relevante & Trabajos \\
\hline \multirow{15}{*}{ Experimentos } & $\begin{array}{l}\text { Imagen por resonancia } \\
\text { magnética funcional (fMRI) }\end{array}$ & $\begin{array}{c}\text { Plassmann et al. (2007), Seymour et al. (2007), Preuschoff et al. } \\
\text { (2008), Engelmann et al. (2009), Halko et al. (2009), Burke et al. } \\
\text { (2010), Miyapuram et al. (2012), Causse et al. (2013), Mullett y } \\
\text { Tunney (2013), Brunnlieb et al. (2016), Suzuki et al. (2016), } \\
\text { Raggetti et al. (2017) }\end{array}$ \\
\hline & $\begin{array}{l}\text { Imagen por resonancia } \\
\text { magnética (MRI) }\end{array}$ & Kalenscher et al. (2010) \\
\hline & $\begin{array}{c}\text { Espectroscopia por } \\
\text { infrarrojo cercano (fNIRS) }\end{array}$ & Shimokawa et al. (2012), Holper et al. (2014) \\
\hline & $\begin{array}{c}\text { Estímulo de corriente } \\
\text { directa transcraneal (tDCS) }\end{array}$ & Zhang (2018) \\
\hline & Muestras de saliva & Coates y Herbert (2008), Sapienza et al. (2009) \\
\hline & $\begin{array}{c}\text { Electroencefalograma } \\
\text { (EEG) }\end{array}$ & Shen et al. (2013), Wang (2015) \\
\hline & Genotipado & Frydman et al. (2011) \\
\hline & $\begin{array}{l}\text { Respuesta galvánica de la } \\
\text { piel (SCR) }\end{array}$ & Lucarelli et al. (2015) \\
\hline & $\begin{array}{l}\text { Método/Modelamiento } \\
\text { computacional }\end{array}$ & Shoji y Kanehiro (2012), Zhu et al. (2012), Saposnik et al. (2019) \\
\hline & $\begin{array}{l}\text { Análisis neurogenético y } \\
\text { neuroquímico }\end{array}$ & Zhong et al. (2009) \\
\hline & \multirow{5}{*}{ Aplicación de pruebas } & Spinella et al. (2007) - Escala de Finanzas personales ejecutivas \\
\hline & & $\begin{array}{c}\text { Spinella et al. (2004) - Escala de Comportamiento del Sistema } \\
\text { Frontal }\end{array}$ \\
\hline & & Kuhnen \& Knutson (2011) - Prueba de creencias \\
\hline & & James (2018) - Interés frente a donaciones caritativas \\
\hline & & Zandstra et al. (2013) - Reclamos verdes \\
\hline \multirow{4}{*}{\multicolumn{2}{|c|}{ Modelamiento }} & $\begin{array}{c}\text { Chiong et al. (2014), Nienhusser \& Oshio (2017) - Análisis } \\
\text { factorial }\end{array}$ \\
\hline & & Prinz et al. (2014) - ANOVA \\
\hline & & Saposnik et al. (2017) - Análisis corte transversal \\
\hline & & Ardalan (2018) - Modelo matemático \\
\hline Rev & visión de literatura & $\begin{array}{l}\text { Bossaerts (2009), Baddeley (2010), Mohr et al. (2010), Reimann \& } \\
\text { Bechara (2010), Bermejo et al. (2011), Camerer (2013), Pulcu et } \\
\text { al. (2013), Tzieropoulos (2013), Apicella et al. (2015), Kolev et al. } \\
\text { (2015), Knecht \& Kenning (2016), Strombach et al. (2016), Mateu } \\
\text { et al. (2017), Erkut et al. (2018), Harris et al. (2018), Brisset y } \\
\text { Jullien (2020), Hu y Shealy (2019), Srivastava et al. (2019) }\end{array}$ \\
\hline
\end{tabular}

Fuente: elaboración propia a partir de Web of Science. 
A partir de la clasificación realizada, es pertinente resaltar que el $57.41 \%$ de los estudios revisados posee los experimentos como enfoque o método de investigación; seguido por un $33.33 \%$ asociado a revisiones de literatura y un $9.26 \%$ relacionado con modelamiento matemático y estadístico. De esta manera, se encuentra que el enfoque de investigación basado en experimentos se erige como uno de los más relevantes en las investigaciones asociadas a la neuroeconomía en el campo de la toma de decisiones financieras.

\section{DISCUSIÓN}

Los resultados presentados evidencian una relación estrecha con un campo específico de la economía del comportamiento denominado finanzas comportamentales. De esta manera, es pertinente complementar los hallazgos obtenidos con una breve descripción de los trabajos de Simon (1989), Kahneman y Tversky (1979) y De Bondt y Thaler (1995) para destacar los siguientes conceptos centrales relevantes: la racionalidad limitada, la teoría prospectiva y los microfundamentos de las finanzas comportamentales. De manera general, la clasificación propuesta en la Tabla 4 puede evidenciar que un concepto transversal al tema central de interés es la racionalidad limitada, que las reflexiones teóricas parten de reflexiones alrededor de la teoría prospectiva y las aplicaciones a la toma de decisiones, finanzas y la conducta están soportadas en los microfundamentos de las finanzas comportamentales. Así cobra sentido abordar estos conceptos para complementar el análisis bibliométrico realizado en aras de tener un panorama más amplio para dar alcance a los objetivos propuestos.

La racionalidad limitada: a partir de lo discutido anteriormente, es posible identificar que uno de los elementos claves del mainstream del análisis económico, ha girado en torno al supuesto de la racionalidad completa de los individuos; sin embargo, dicho supuesto ha generado diversas inconsistencias frente a las formulaciones teóricas y los escenarios reales de actuación de estos, lo cual derivó en el surgimiento de la economía del comportamiento como una rama de la economía que pretende analizar la conducta de los individuos en escenarios/experimentos sin supuestos/juicios a priori.

A partir de esta crítica, Simon (1989) soporta su análisis en la teoría de la racionalidad limitada, donde la estructura de razonamiento de los individuos busca alcanzar mínimos niveles de satisfacción para luego ajustar la elección y elevar su satisfacción acorde a las condiciones de su entorno (tomando como punto de partida la biología). Especialmente, analiza la naturaleza y límites de la razón humana, al enfatizar en tres ideas centrales: i) la relación que tiene la razón con la intuición y la emoción, ii) la analogía entre adaptación racional y evolución y, iii) las implicaciones de la racionalidad limitada para la operación de las instituciones políticas y sociales. De manera específica, Simon (1989) cuestiona el optimismo que se tiene en algunas ciencias sociales al afirmar que los individuos son lo suficientemente racionales para resolver todos los problemas, destacando que la comprensión de los mismos no tienen en cuenta los siguientes elementos: 1) se pretende plantear leyes infalibles a partir de hechos específicos (y no generales), lo cual lleva a errores en los planteamientos; 2) los análisis están basados en diversas suposiciones teóricas que facilitan su comprensión (estrechando aún más el panorama de la realidad) y; 3) ante esta limitación del proceso de razonamiento para postular conclusiones generales y consistentes, se adiciona el agravante que, en muchas ocasiones, la razón se altera por valores, hechos y emociones. 
Con el fin de comprender de una forma más cercana a la realidad la conducta humana, Simon (1989) desarrolla el modelo de la racionalidad limitada, basado en los siguientes hechos: 1) las decisiones de los individuos no son elecciones que abarcan grandes áreas de su vida, sino que tienden a ser relativamente independientes de otras dimensiones de la vida; 2 ) no se calcula de manera rigurosa las consecuencias de una decisión para el futuro (y menos las distribuciones de probabilidad dependientes de la alternativa escogida), 3) los individuos, una vez que toman una decisión, dejan de analizar las alternativas posibles y van tomando un rumbo que va sesgando su comportamiento y, 4) a pesar de las asimetrías de información, los individuos buscan recolectar la mayor información posible para la toma de decisiones. Así, este modelo conductual es consciente de las interconexiones entre variables que afectan el mundo, pero se enfoca en las consideraciones que significativamente predominan y afectan al individuo; en otras palabras, se caracteriza porque el ser humano toma sus decisiones basado en su adaptación, comprensión y priorización de los problemas del entorno; restringido por el orden que debe dar a sus problemas, las emociones que lo pueden conducir a un rumbo determinado y por la información que pueda obtener del medio ambiente.

La teoría prospectiva: esta teoría desarrollada por Kahneman y Tversky (1979) cuestiona los supuestos de la teoría de la utilidad esperada propuesta por Von Neumann y Morgenstern (1944), considerado como uno de los paradigmas en la toma de decisiones financieras, mediante la realización de experimentos simples que evidencian la presencia de los siguientes efectos: i) efecto certeza: relacionado con el hecho que se sobrestiman resultados que se consideran ciertos en relación con los resultados que son meramente probables; ii) efecto reflejo: que evidencia que la aversión al riesgo (que tiende a ser cóncava) en escenarios de ganancias no se refleja de igual manera en escenarios de pérdida, al evidenciarse la búsqueda de riesgo en terrenos negativos (funciones convexas); iii) efecto aislamiento: que resalta que las personas a menudo ignoran los componentes que comparten alternativas y se concentran únicamente en los componentes que los distinguen. A partir de lo anterior, la presencia de los efectos mencionados viola los axiomas de transitividad, reflexividad e independencia de las preferencias asumidas por la teoría de la utilidad esperada; de esta manera, la racionalidad limitada de los individuos juega un papel esencial en los resultados de los experimentos propuestos y dificulta que la teoría propuesta por Von Neumann y Morgenstern (1944) sea un paradigma sólido en el análisis económico.

A partir de lo anterior, Kahneman y Tversky (1979) proponen una 'teoría prospectiva', la cual se desarrolla para prospectos simples con resultados monetarios y probabilidades claras donde se distinguen dos fases en el proceso de decisión de los individuos: una fase temprana de edición (que contempla una serie de procesos de codificación, combinación, segregación y simplificación) y una fase posterior de evaluación; proceso que es coherente con los postulados de las ciencias cognitivas que resalta la complejidad de la toma de decisiones. En este sentido, la teoría mencionada analiza el proceso de toma de decisiones de los individuos, en escenarios de riesgo, de una manera más lógica y ajustada a sus procesos cognitivos, destacando los siguientes puntos clave: i) las actitudes hacia el riesgo se determinan conjuntamente, y no únicamente por la función de utilidad; ii) es instructivo examinar las condiciones bajo las cuales se espera que ocurra la aversión al riesgo o la búsqueda del riesgo; iii) hay situaciones en las que las ganancias y las pérdidas se codifican en relación con una expectativa o nivel de aspiración que difiere del statu quo; iv) puede surgir una discrepancia entre el punto de referencia y la posición del activo actual debido a los cambios recientes en la riqueza a los que todavía no se ha adaptado el individuo; v) el lapso de tiempo y los juicios emitidos (asociados a influencia social) inciden en el proceso de toma de decisiones. 
Microfundamentos de las finanzas comportamentales: en la misma lógica de los aportes de Simon (1989), Kahnemany y Tversky (1979) y otros autores de las finanzas comportamentales, De Bondt y Thaler (1995) realizan una detallada revisión de literatura donde destacan que el proceso de toma de decisiones y elaboración de predicciones de parte de personas, firmas y mercados están sujetos a la presencia de la racionalidad limitada de los individuos, los cuales están caracterizados por los siguientes fenómenos de comportamiento resumidos en la Tabla 6.

Tabla 6. Microfundamentos de las finanzas comportamentales

Table 6. Microfoundations of behavioral finance

Exceso de confianza

Heurística de representatividad

Aversión al riesgo, encuadre (framing) y contabilidad mental

Arrepentimiento, responsabilidad y prudencia

Asociado al hecho que los individuos sobreestiman la fiabilidad de su conocimiento, de sus habilidades y de sus predicciones.

Relacionado con la búsqueda de «atajos mentales» donde los individuos evalúan la probabilidad de un evento incierto, o una muestra, según la similitud con las propiedades esenciales para su población matriz o el reflejo de las características sobresalientes del proceso por el cual se genera, pudiendo llevar a errores sistemáticos.

Implica que la aversión/propensión al riesgo depende de los cambios en la riqueza, ante el hecho que los cambios negativos se ponderan más que las ganancias. Además, dicha aversión implica que la toma de decisiones es sensible al marco o delimitación de opciones de acción, donde las personas llevan una 'contabilidad mental' de dichas opciones y procesos.

Ilustra el efecto de la influencia social sobre las decisiones de los individuos, especialmente evidente en fenómenos de 'comportamiento de manada/rebaño' donde sienten presión por seguir una tendencia/patrón marcado por dicha influencia.

Evidencia diferentes sensaciones ex post sobre decisiones que condujeron a malos resultados, lo cual es de interés de estudio en la medida en que los tomadores de decisiones tomen medidas para evitar el arrepentimiento mediante estrategias como transferir la responsabilidad de una decisión a otra persona (vincular a un agente) o siguiendo normas sociales y legales «estándar» desde el punto de vista de la prudencia.

Fuente: elaboración propia a partir de De Bondt y Thaler (1995).

A partir del anterior esquema, los autores afirman que la comprensión de las finanzas comportamentales complementa y amplía los paradigmas de las finanzas modernas en el sentido en que, el uso de diversos métodos de investigación y el apoyo en diferentes disciplinas, facilita la observación cuidadosa de lo que realmente hacen las personas, sin necesidad de proponer supuestos artificiales que se alejan de la realidad y no permiten plantear principios de comportamiento generales que se apliquen en múltiples contextos. De esta manera, el análisis de variables relevantes en el sistema económico como decisiones bajo escenarios de riesgo, expectativas y fijación de precios es más potente en la medida en que el instrumental matemático y analítico de la economía ortodoxa es combinado con el esquema analítico y experimental de la economía y finanzas comportamentales.

Para finalizar con esta sección es importante resaltar que el campo de investigación de las finanzas comportamentales, $y$, de manera más amplia, la economía experimental, es indispensable para la 
problemática de investigación propuesta en el sentido que brinda el marco analítico para comprender la conducta y proceso de toma de decisiones de los individuos, apoyado en una diversidad de ejemplos de experimentos y aplicaciones, que facilitan conectar los principales modelos microeconómicos con la óptica realista de la racionalidad limitada y la complejidad de la toma de decisiones financieras.

\section{CONCLUSIONES}

La comprensión de los avances en neuroeconomía, tema de investigación que ha cobrado relevancia en años recientes, parten de la economía del comportamiento como campo de estudio que suscitó la comprensión de la conducta de los agentes económicos, especialmente la toma de decisiones, apoyado de las neurociencias cognitivas aplicadas. Por otra parte, la revisión de literatura realizada permite identificar los principales trabajos sobre neuroeconomía aplicados a la toma de decisiones financieras, presentando un amplio abanico de posibilidades desde su principal enfoque como lo son los experimentos, a la luz de diferentes equipos de análisis neurológico (resonancia magnética, electroencefalograma, entre otros). En esta vía se encuentran diversos aspectos susceptibles de analizarse en la toma de decisiones financieras como lo son la aversión al riesgo, la gestión de finanzas y la estructura de incentivos. De otro lado, los principales expertos sobre este tema se ubican en Estados Unidos y Europa.

De esta manera, el ejercicio realizado constituye un insumo exploratorio valioso para investigadores de la región a la hora de entender el estado de la literatura relacionada con la toma de decisiones financieras en el campo de la neuroeconomía. Así, el ejercicio realizado permite identificar la oportunidad de ahondar en este tema de investigación dado que se registran pocas publicaciones para el caso latinoamericano, según los estándares de alta calidad en publicaciones científicas; teniendo en cuenta que los principales estudios se encuentran publicados en las principales revistas de neurociencia del mundo. En este sentido, se identifica una línea de investigación interesante por desarrollar en la región con la aplicación de técnicas empíricas y experimentales identificadas en la revisión, donde las reflexiones teóricas van siendo robustecidas por técnicas empíricas desde diferentes disciplinas para una mejor comprensión del comportamiento y decisiones de los seres humanos. De esta manera, esta revisión pretende ser referente de subsiguientes investigaciones relacionadas en América Latina.

\section{CONFLICTOS DE INTERÉS}

Los autores declaran que no presentan conflictos de interés financiero, profesional o personal que pueda influir de forma inapropiada en los resultados obtenidos o las interpretaciones propuestas.

\section{CONTRIBUCIÓN DE AUTORES}

Para el desarrollo de este proyecto todos los autores han realizado una contribución significativa especificada a continuación: 
Daniel Osorio-Barreto: análisis bibliométrico y reflexión a la luz de la teoría económica.

Daniel Alfredo Landínez-Martínez: reflexión y análisis desde la neuropsicología.

Juan Carlos Chica-Mesa: reflexión y análisis desde las finanzas comportamentales.

\section{REFERENCIAS}

Angner, E., Loewenstein, G. (2012). Behavioral Economics. In U. Mäki (Ed.). Philosophy of Economics (pp. 641-689). Elsevier.

Apicella, C. L., Carré, J. M., Dreber, A. (2015). Testosterone and Economic Risk Taking: A Review. Adaptive Human Behavior and Physiology, v. 1, n. 3, 358-385. https://doi.org/10.1007/s40750-014-0020-2

Ardalan, K. (2018). Neurofinance versus the efficient markets hypothesis. Global Finance Journal, v. 35, 170-176. https://doi.org/10.1016/i.gfj.2017.10.005

Aria, M., Cuccurullo, C. (2017). bibliometrix: An R-tool for comprehensive science mapping analysis. Journal of Informetrics, v. 11, n. 4, 959-975. https://doi.org/10.1016/i.joi.2017.08.007

Baddeley, M. (2010). Herding, social influence and economic decision-making: socio-psychological and neuroscientific analyses. Philosophical Transactions of the Royal Society B: Biological Sciences, v. 365, n. 1538, 281-290. https://doi.org/10.1098/rstb.2009.0169

Bermejo, P. E., Dorado, R., Zea-Sevilla, M. A., Sánchez Menéndez, V. (2011). Neuroanatomía de las decisiones financieras. Neurología, v. 26, n. 3, 173-181. https://doi.org/10.1016/i.nrl.2010.09.015

Bigorra, A., Garolera, M., Guijarro, S., \& Hervas, A. (2016). Impact of working memory training on hot executive functions (decision-making and theory of mind) in children with ADHD: a randomized controlled trial. Neuropsychiatry, v. 6, n. 5, 251-263. https://doi.org/10.4172/Neuropsychiatry.1000147

Bossaerts, P. (2009). What Decision Neuroscience Teaches Us About Financial Decision Making. Annual Review of Financial Economics, v. 1, n. 1, 383-404. https://doi.org/10.1146/annurev.financial.102708.141514

Brisset, N., Jullien, D. (2020). The model (also) in the world: extending the sociological theory of fields to economic models. Journal of Economic Methodology, v. 27, n. 2, 130-145. https://doi.org/10.1080/1350178X.2019.1680857 
Brunnlieb, C., Nave, G., Camerer, C. F., Schosser, S., Vogt, B., Münte, T. F., Heldmann, M. (2016). Vasopressin increases human risky cooperative behavior. Proceedings of the National Academy of Sciences of the United States of America, v. 113, n. 8, 2051-2056. https://doi.org/10.1073/pnas.1518825113

Burke, C., Tobler, P. N., Schultz, W., Baddeley, M. (2010). Striatal BOLD response reflects the impact of herd information on financial decisions. Frontiers in Human Neuroscience, v. 4, n. 48, 1-11. https://doi.org/10.3389/fnhum.2010.00048

Camerer, C., Loewenstein, G., Prelec, D. (2005). Neuroeconomics: How neuroscience can inform economics. Journal of Economic Literature, v. 43, n. 1, 9-64. https://doi.org/10.1257/0022051053737843

Camerer, C. F. (2013). Goals, Methods, and Progress in Neuroeconomics. Annual Review of Economics, v. 5, 425-455. https://doi.org/10.1146/annurev-economics-082012-123040

Carminati, L. (2020). Behavioural Economics and Human Decision Making: Instances from the Health Care System. Health Policy, v. 124, n. 6, 659-664. https://doi.org/10.1016/i.healthpol.2020.03.012

Causse, M., Dehais, F., Péran, P., Sabatini, U., Pastor, J. (2013). The effects of emotion on pilot decision-making: A neuroergonomic approach to aviation safety. Transportation Research Part C: Emerging Technologies, v. 33, 272-281. https://doi.org/10.1016/i.trc.2012.04.005

Chiong, W., Hsu, M., Wudka, D., Miller, B. L., Rosen, H. J. (2014). Financial errors in dementia: Testing a neuroeconomic conceptual framework. Neurocase, v. 20, n. 4, 389-396. https://doi.org/10.1080/13554794.2013.770886

Coates, J. M., Herbert, J. (2008). Endogenous steroids and financial risk taking on a London trading floor. Proceedings of the National Academy of Sciences of the United States of America, v. 105, n. 1, 6167-6172. https://doi.org/10.1073/pnas.0704025105

De Bondt, W. F., Thaler, R. H. (1995). Financial decision-making in markets and firms: A behavioral perspective. Handbooks in operations research and management science, v. 9, 385-410. https://doi.org/10.1016/S0927-0507(05)80057-X

Engelmann, J. B., Capra, C. M., Noussair, C., Berns, G. S. (2009). Expert financial advice neurobiologically "offloads" financial decision-making under risk. PLOS ONE, v. 4, n. 3, 1-14. https://doi.org/10.1371/journal.pone.0004957

Erkut, B., Kaya, T., Lehmann-Waffenschmidt, M., Mahendru, M., Sharma, G. D., Srivastava, A. K., Srivastava, M. (2018). A fresh look on financial decision-making from the plasticity perspective. International Journal of Ethics and Systems, v. 34, n. 4, 426-441.

https://doi.org/10.1108/IJOES-02-2018-0022 
Fairchild, G., van Goozen, S. H. M., Stollery, S. J., Aitken, M. R. F., Savage, J., Moore, S. C., Goodyer, I. M. (2009). Decision Making and Executive Function in Male Adolescents with Early-Onset or Adolescence-Onset Conduct Disorder and Control Subjects. Biological Psychiatry, v. 66, n. 2, 162-168. https://doi.org/10.1016/j.biopsych.2009.02.024

Fatima, S., Khan, M., Rosselli, M., Ardila, A. (2020). Age, executive functioning, and decision-making styles in adults: a moderated mediation model. Aging, Neuropsychology, and Cognition, v. 27, n. 3, 338-350. https://doi.org/10.1080/13825585.2019.1614142

Frydman, C., Camerer, C., Bossaerts, P., Rangel, A. (2011). MAOA-L carriers are better at making optimal financial decisions under risk. Proceedings of the Royal Society B: Biological Sciences, v. 278, n. 1714, 2053-2059. https://doi.org/10.1098/rspb.2010.2304

Glimcher, P. W. (2004). Decision, Uncertainty, and the Brain: The Science of Neuroeconomics. MIT Press.

Halko, M. L., Hlushchuk, Y., Hari, R., Schürmann, M. (2009). Competing with peers: Mentalizingrelated brain activity reflects what is at stake. Neurolmage, v. 46, n. 2, 542-548. https://doi.org/10.1016/i.neuroimage.2009.01.063

Harris, J. M., Ciorciari, J., Gountas, J. (2018). Public health social media communications and consumer neuroscience. Cogent Psychology, v. 5, n. 1, 1-13. https://doi.org/10.1080/23311908.2018.1434058

Holper, L., Wolf, M., Tobler, P. N. (2014). Comparison of functional near-infrared spectroscopy and electrodermal activity in assessing objective versus subjective risk during risky financial decisions. Neurolmage, v. 84, 833-842. https://doi.org/10.1016/j.neuroimage.2013.09.047

Hsu, M., Bhatt, M., Adolphs, R., Tranel, D., Camerer, C. F. (2005). Neural systems responding to degrees of uncertainty in human decision-making. Science, v. 310, n. 5754, 1680-1683. https://doi.org/10.1126/science.1115327

Hu, M., Shealy, T. (2019). Application of functional near-infrared spectroscopy to measure engineering decision-making and design cognition: literature review and synthesis of methods. Journal of Computing in Civil Engineering, v. 33, n. 6, 04019034. https://doi.org/10.1061/(ASCE)CP.1943-5487.0000848

James, R. N. (2018). Describing complex charitable giving instruments: Experimental tests of technical finance terms and tax benefits. Nonprofit Management and Leadership, v. 28, n. 4, 437-452. https://doi.org/10.1002/nml.21302

Kahneman, D., Tversky, A. (1979). Prospect Theory: An Analysis of Decision under Risk. Econometrica, v. 47, n. 2, 263-292. https://doi.org/10.2307/1914185 
Kalenscher, T., Tobler, P. N., Huijbers, W., Daselaar, S. M., Pennartz, C. M. A. (2010). Neural signatures of intransitive preferences. Frontiers in Human Neuroscience, v. 4, n. 49, 1-14. https://doi.org/10.3389/fnhum.2010.00049

Katona, G. (1946). Psychological Analysis of Business Decisions and Expectations. The American Economic Review, v. 36, n. 1, 44-62. https://www.jstor.org/stable/1802255

Katona, G. (1953). Rational Behavior and economic Behavior. Psychological Review, v. 60, n. 5, 307318. https://doi.org/10.1037/h0060640

Knecht, S., Kenning, P. (2016). Changing health behavior motivation from I-must to I-want. In V. Walsh (Ed.). Motivation: Theory, Neurobiology and Applications (pp. 427-438).

Knutson, B., Rick, S., Wimmer, G. E., Prelec, D., Loewenstein, G. (2007). Neural predictors of purchases. Neuron, v. 53, n. 1, 147-156. https://doi.org/10.1016/i.neuron.2006.11.010

Kolev, B., Njegovanovic, A., Cosic K. (2015). Neuroeconomics: A contemporary scientific field and innovative approach as a significant factor of economic development. Casopis Za Ekonomiju I Trzisne Komunikacije, v. 5, n. 2, 278-296. URL

Kuhnen, C. M., Knutson, B. (2005). The neural basis of financial risk taking. Neuron, v. 47, n. 5, 763770. https://doi.org/10.1016/j.neuron.2005.08.008

Kuhnen, C. M., Knutson, B. (2011). The Influence of Affect on Beliefs, Preferences, and Financial Decisions. Journal of Financial and Quantitative Analysis, v. 46, n. 3, 605-626. https://doi.org/10.1017/S0022109011000123

Loewenstein, G. (1996). Out of Control: Visceral Influence on Behavior. Organizational Behavior and Human Decision Processes, v. 65, n. 3, 272-292. https://doi.org/10.1006/obhd.1996.0028

Lucarelli, C., Uberti, P., Brighetti, G., Maggi, M. (2015). Risky choices and emotion-based learning. Journal of Economic Psychology, v. 49, 59-73. https://doi.org/10.1016/j.joep.2015.04.004

Martin, K. A., Sbicca, A. (2021). Decisões financeiras e o uso de aplicativos: um estudo à luz da economia comportamental. RGO - Revista Gestão Organizacional, v. 14, n. 2, 207-228. https://doi.org/10.22277/rgo.v14i2.5443

Mateu, G., Monzani, L., Muñoz Navarro, R. (2017). The role of the brain in financial decisions: A review of the neuroeconomics field. Mètode Science Studies Journal, n. 8, 6-15. https://doi.org/10.7203/metode.8.6923

McClure, S. M., Laibson, D. I., Loewenstein, G., Cohen, J. D. (2004). Separate neural systems value immediate and delayed monetary rewards. Science, v. 306, n. 5695, 503-507. https://doi.org/10.1126/science.1100907 
Miyapuram, K. P., Tobler, P. N., Gregorios-Pippas, L., \& Schultz, W. (2012). BOLD responses in reward regions to hypothetical and imaginary monetary rewards. Neuroimage, v. 59, n. 2, 16921699. https://doi.org/10.1016/j.neuroimage.2011.09.029

Mohr, P. N. C., Li, S. C., Heekeren, H. R. (2010). Neuroeconomics and aging: Neuromodulation of economic decision making in old age. Neuroscience and Biobehavioral Reviews, v. 34, n. 5, 678-688. https://doi.org/10.1016/i.neubiorev.2009.05.010

Mullett, T. L., Tunney, R. J. (2013). Value representations by rank order in a distributed network of varying context dependency. Brain and Cognition, v. 82, n. 1, 76-83. https://doi.org/10.1016/j.bandc.2013.02.010

Nienhusser, H. K., Oshio, T. (2017). High school students' accuracy in estimating the cost of college: A proposed methodological approach and differences among racial/ethnic groups and college financial-related factors. Research in Higher Education, v. 58, n. 7, 723-745. https://doi.org/10.1007/s11162-017-9447-1

Plassmann, H., O'Doherty, J., Rangel, A. (2007). Orbitofrontal Cortex Encodes Willingness to Pay in Everyday Economic Transactions. The Journal of Neuroscience, v. 27, n. 3, 9984-9988. https://doi.org/10.1523/JNEUROSCI.2131-07.2007

Preuschoff, K., Quartz, S., Bossaerts, P. (2008). Markowitz in the brain? Revue D'économie Politique, v. 118, n. 1, 75-95. https://doi.org/10.3917/redp.181.0075

Prinz, S., Gründer, G., Hilgers, R. D., Holtemöller, O., Vernaleken, I. (2014). Impact of personal economic environment and personality factors on individual financial decision making. Frontiers in Psychology, v. 5(MAR), 1-11. https://doi.org/10.3389/fpsyg.2014.00158

Pulcu, E., Zahn, R., Elliott, R. (2013). The role of self-blaming moral emotions in major depression and their impact on social-economical decision making. Frontiers in Psychology, v. 4, n. 310, 1-17. https://doi.org/10.3389/fpsyg.2013.00310

Raggetti, G., Ceravolo, M. G., Fattobene, L., Di Dio, C. (2017). Neural Correlates of Direct Access Trading in a Real Stock Market: An fMRI Investigation. Frontiers in Neuroscience, v. 11, 1-14. https://doi.org/10.3389/fnins.2017.00536

Rangel, A., Camerer, C., \& Montague, P. R. (2008). A framework for studying the neurobiology of value-based decision making. Nature reviews neuroscience, v. 9, n. 7, 545-556. https://doi.org/10.1038/nrn2357

Reimann, M., Bechara, A. (2010). The somatic marker framework as a neurological theory of decisionmaking: Review, conceptual comparisons, and future neuroeconomics research. Journal of Economic Psychology, v. 31, n. 5, 767-776. https://doi.org/10.1016/j.joep.2010.03.002 
Robledo, S., Osorio, G., López, C. (2014). Networking en pequeña empresa: una revisión bibliográfica utilizando la teoría de grafos. Revista Vínculos, v. 11, n. 2, 6-16.

https://doi.org/10.14483/2322939X.9664

Sanfey, A. G., Rilling, J. K., Aronson, J. A., Nystrom, L. E., Cohen, J. D. (2003). The neural basis of economic decision-making in the ultimatum game. Science, v. 300, n. 5626, 1755-1758. https://doi.org/10.1126/science.1082976

Sapienza, P., Zingales, L., Maestripieri, D. (2009). Gender differences in financial risk aversion and career choices are affected by testosterone. Proceedings of the National Academy of Sciences of the United States of America, v. 106, n. 36, 15268-15273. https://doi.org/10.1073/pnas.0907352106

Saposnik, G., Sempere, A. P., Prefasi, D., Selchen, D., Ruff, C. C., Maurino, J., Tobler, P. N. (2017). Decision-making in Multiple Sclerosis: The Role of Aversion to Ambiguity for Therapeutic Inertia among Neurologists (DIScUTIR MS). Frontiers in Neurology, v. 8, 1-8. https://doi.org/10.3389/fneur.2017.00065

Saposnik, G., Oh, J., Terzaghi, M. A., Kostyrko, P., Bakdache, F., Montoya, A., Blessing, N. R. J., Nisembaum, R., Ruff, C. C., Tobler, P. N. (2019). Emotional expressions associated with therapeutic inertia in multiple sclerosis care. Multiple sclerosis and related disorders, v. 34, 17-28. https://doi.org/10.1016/j.msard.2019.05.029

Satterfield, B. C., Killgore, W. D. S. (2019). Sleep loss, executive function, and decision-making. In Sleep and Health (pp. 339-358). Elsevier. https://doi.org/10.1016/B978-0-12-815373-4.00026-5

Schultz, W., Dayan, P., Montague, P. R. (1997). A neural substrate of prediction and reward. Science, v. 275, n. 5306, 1593-1599. https://doi.org/10.1126/science.275.5306.1593

Seymour, B., Daw, N., Dayan, P., Singer, T., Dolan, R. (2007). Differential encoding of losses and gains in the human striatum. The Journal of Neuroscience, v. 27, n. 18, 4826-4831. https://doi.org/10.1523/JNEUROSCI.0400-07.2007

Shen, Q., Jin, J., Ma, Q. (2013). The sweet side of inequality: How advantageous status modulates empathic response to others' gains and losses. Behavioural Brain Research, v. 256, 609-617. https://doi.org/10.1016/j.bbr.2013.08.043

Shimokawa, T., Kinoshita, K., Miyagawa, K., Misawa, T. (2012). A brain information-aided intelligent investment system. Decision Support Systems, v. 54, n. 1, 336-344. https://doi.org/10.1016/i.dss.2012.05.041

Shoji, I., Kanehiro, S. (2012). Intertemporal dynamic choice under myopia for reward and different risk tolerances. Economic Theory, v. 50, n. 1, 85-98. https://doi.org/10.1007/s00199-010-0534-1 
Simon, H. A. (1955). A Behavioral Model of Rational Choice. The Quarterly Journal of Economics, v. 69, n. 1, 99-118. https://doi.org/10.2307/1884852

Simon, H. A. (1989). Naturaleza y límites de la razón humana. Fondo de Cultura Económica.

Spinella, M., Yang, B., Lester, D. (2004). Prefrontal System Dysfunction and Credit Card Debt. International Journal of Neuroscience, v. 114, n. 10, 1323-1332. https://doi.org/10.1080/00207450490476011

Spinella, M., Yang, B., Lester, D. (2007). Development of the Executive Personal Finance Scale. International Journal of Neuroscience, v. 117, n. 3, 301-313. https://doi.org/10.1080/00207450500534043

Srivastava, M., Sharma, G. D., Srivastava, A. K. (2019). Human brain and financial behavior: a neurofinance perspective. International Journal of Ethics and Systems, v. 25, n. 4, 485-503. https://doi.org/10.1108/IJOES-02-2019-0036

Strombach, T., Strang, S., Park, S. Q., Kenning, P. (2016). Common and distinctive approaches to motivation in different disciplines. In V. Walsh (Ed.). Motivation: Theory, Neurobiology and Applications (pp. 3-23).

Suzuki, S., Jensen, E. L. S., Bossaerts, P., Doherty, J. P. O. (2016). Behavioral contagion during learning about another agent's risk-preferences acts on the neural representation of decision-risk. Proceedings of the National Academy of Sciences of the United States of America, v. 113, n. 14, 3755-3760. https://doi.org/10.1073/pnas.1600092113

Thaler, R. (1980). Toward a positive theory of consumer choice. Journal of Economic Behavior \& Organization, v. 1, n. 1, 39-60. https://doi.org/10.1016/0167-2681(80)90051-7

Tversky, A., Kahneman, D. (1991). Loss Aversion in Riskless Choice: A Reference-Dependent Model. The Quarterly Journal of Economics, v. 106, n. 4, 1039-1061. https://doi.org/10.2307/2937956

Tzieropoulos, H. (2013). The Trust Game in neuroscience: A short review. Social Neuroscience, v. 8, n. 5, 407-416. https://doi.org/10.1080/17470919.2013.832375

Von Neumann, J., Morgenstern, O. (1944). Theory of Games and Economic Behavior. Princeton University Press.

Wang, C., Vieito, J. P., Ma, Q. (2015). A Neuroeconomics Analysis of Investment Process with Money Flow Information: The Error-Related Negativity. Computational Intelligence and Neuroscience, v. 2015, 1-12. https://doi.org/10.1155/2015/701237

Witte, D. K., López-Torres, L. (2017). Efficiency in education: a review of literature and a way forward. Journal of the Operational Research Society, v. 68, n. 4, 339-363. https://doi.org/10.1057/jors.2015.92 
Yang, X., Gao, M., Shi, J., Ye, H., Chen, S. (2017). Modulating the Activity of the DLPFC and OFC Has Distinct Effects on Risk and Ambiguity Decision-Making: A tDCS Study. Frontiers in Psychology, v. 8, 1417. https://doi.org/10.3389/fpsyg.2017.01417

Zandstra, E. H., Miyapuram, K. P., Tobler, P. N. (2013). Understanding consumer decisions using behavioral economics. In V. S. C. Pammi \& N. Srinivasan (Eds.). Progress in Brain Research (Vol. 202, pp. 197-211). Elsevier B.V. https://doi.org/10.1016/B978-0-444-62604-2.00012-5

Zegarra-Valdivia, J. A., Chino-Vilca, B. N. (2018). Social cognition and executive function impairment in young women with anorexia nervosa. Clínica y Salud, v. 29, n. 3, 107-113. https://doi.org/10.5093/clysa2018a16

Zhang, R. (2018). Research on brand trust and financing risk preference of E-commerce based on neuroeconomic experiment. NeuroQuantology, v. 16, n. 4, 101-106. https://doi.org/10.14704/nq.2018.16.4.1215

Zhong, S., Israel, S., Xue, H., Ebstein, R. P., Chew, S. H. (2009). Monoamine oxidase A gene (MAOA) associated with attitude towards longshot risks. PLOS ONE, v. 4, n. 12, 1-4. https://doi.org/10.1371/journal.pone.0008516

Zhu, L., Walsh, D., Hsu, M. (2012). Neuroeconomic measures of social decision-making across the lifespan. Frontiers in Neuroscience, v. 6, 1-7. https://doi.org/10.3389/fnins.2012.00128 